\title{
Prosthodontic Rehabilitation of Cleft Lip and Palate with Presurgical Nasoalveolar Molding
}

Himani Jain ${ }^{1} \quad$ Manjit Kumar $^{1} \quad$ Aquib Mughal $^{1} \quad$ Sumit Katoch ${ }^{1} \quad$ Navjot Kaur ${ }^{1}$
Address for correspondence Himani Jain, BDS, Department of Prosthodontics, Bhojia Dental College and Hospital, Baddi 173205 , Distt. Solan, Himachal Pradesh, India (e-mail: himani.ruler@gmail.com).
Abstract
Keywords
- cleft lip
- cleft palate
- nasoalveolar molding

The purpose of this article is to illustrate the fabrication process of presurgical nasoalveolar molding (PNAM) prosthesis. Growth of alveolar ridges, lips, and nose is initiated and directed by PNAM prosthesis at the presurgical time. Presurgical nasoalveolar molding helps reduce the future complications of lip and nasal surgery. Because of use of this appliance, there are minimum tension and minimum scar formation due to repair of soft tissue and cartilaginous deformity.

\section{Introduction}

One of the congenital anomalies of middle third of the face is cleft lip and palate. There is significant improvement in quality of repair of cleft lip and palate in recent era. ${ }^{1}$ The basic norms of any treatment related to cleft lip and cleft palate is to restore normal anatomy. ${ }^{2}$ Presurgical nasoalveolar molding was introduced to get better results. ${ }^{3}$ There is active molding and positioning of soft tissue around cleft, which reduced not only cleft size but also deformed soft tissue and cartilage in cleft nose.

\section{Impression Technique}

Parents should be thoroughly informed about the procedure. At the first week of birth, primary impression of the intraoral cleft is made using heavy-body silicone impression material (Zhermack, Badia Polesine (Rovigo) Italy). Infant should be fully awake, and the procedure of impression making is done in the clinical setup that can treat airway emergencies, if at all happened. ${ }^{4,5}$

During impression procedure, position of infant should be upside down and tray is inserted into the patient's mouth to avoid ingress of material into the throat and to allow fluids to drain out of the mouth. Once impression material is set, it is removed and thorough inspection is done for the details of the landmarks achieved (-Fig. 1).

\section{Prosthesis Fabrication}

Vernier caliper is used to measure the level of alveolus on the cast. To get approximate contour and topography of an intact

received

September 24, 2018 accepted after revision

December 7, 2018

published online

January 22, 2019 alveolus, wax is filled in the cleft region. ${ }^{1}$ Cold mold seal is applied as separating media, and molding plate is fabricated on the dental stone cast. Self-cure polymethyl methacrylate (DPI, Mumbai, India) is used to fabricate molding plate. A layer of soft liner (Mollosil Detax, Ettlingen, Germany) is used. A care should be taken to ensure smooth finishing and polishing of the prosthesis that directly comes in contact of labial frenum and tongue, so that these should not cause any ulceration (-Fig. 2).

\section{Prosthesis Insertion}

Care should be taken to insert the prosthesis in infant's oral cavity for sometime. Parents are instructed to keep the prosthesis in oral cavity except for cleaning purpose. The infant may take some time to adjust to prosthesis. Surgical tapes and orthodontic elastics are used extraorally to secure the prosthesis in the place. Tapes should be changed daily.

\section{Prosthesis Adjustment}

Every time the patient is recalled after 1 week to see the alveolar segment going to desired position, intraoral examination is done to check any soreness or ulceration. The prosthesis is adjusted by grinding the hard polymethylmethacrylate, and soft liner is added every time. The grinding of hard acrylic should not be done more than $1 \mathrm{~mm}$. After 1 month of weekly recalled visits, the intra-alveolar distance is measured to see that alveolar segment is directed to its final and desired position. ${ }^{4}$

Pradesh University
(C2018 Bhojia Dental College and

License terms

Hospital affiliated to Himachal

$10.1055 / \mathrm{s}-0039-1677778$

ISSN 2321-1482. 


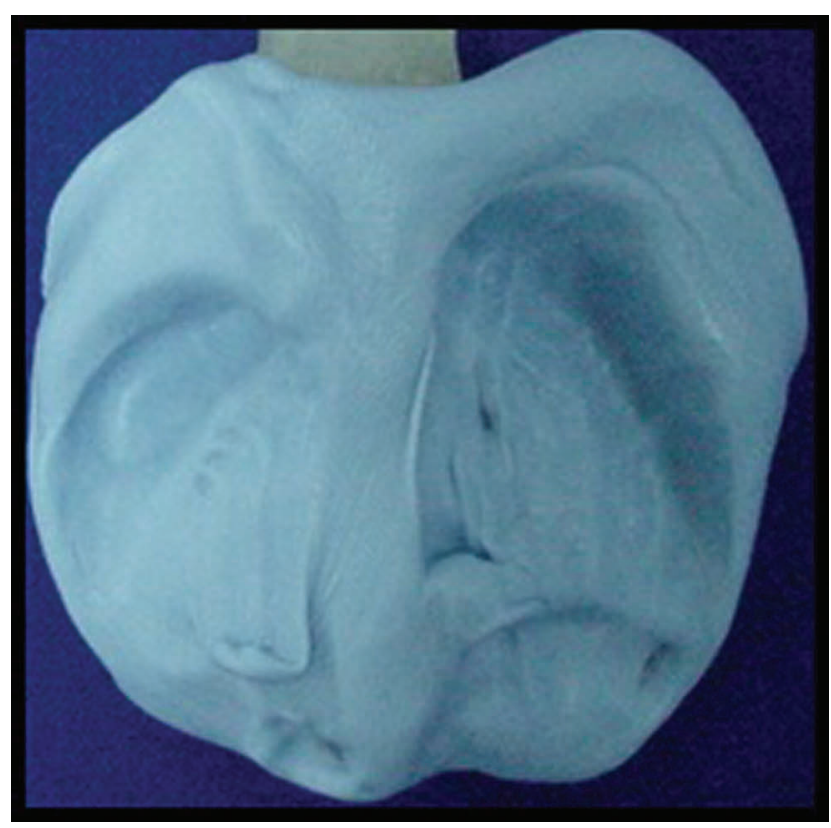

Fig. 1 Impression of patient.

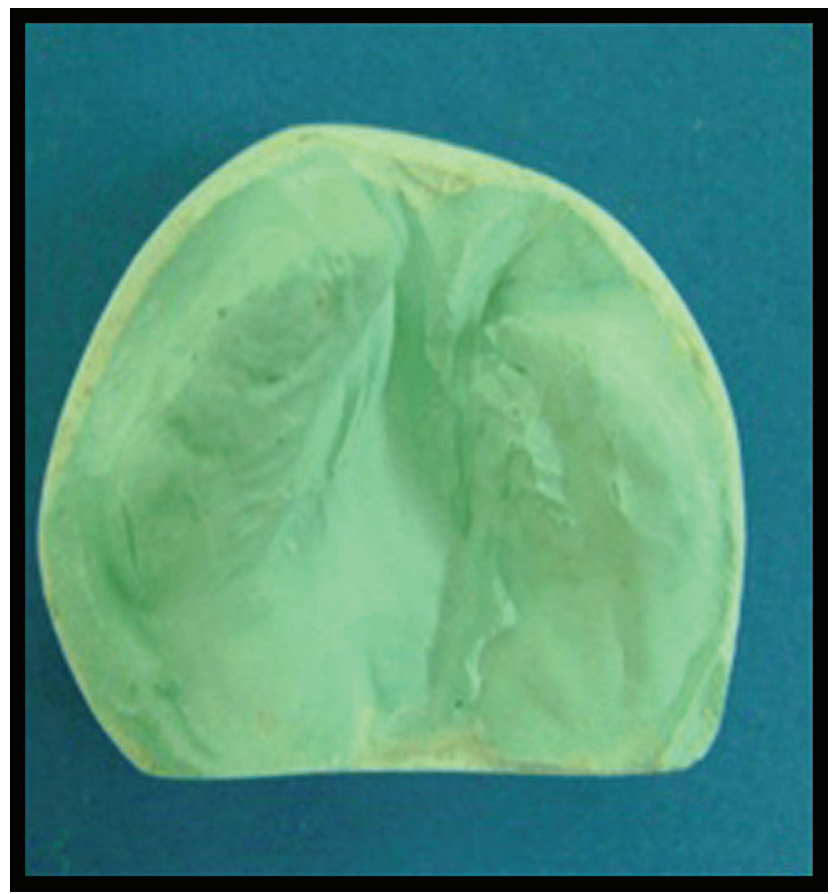

Fig. 2 Stone plaster working cast.

\section{Nasal Stent}

Once the alveolar gap reduces to $5 \mathrm{~mm}$, the nasal component of the prosthesis is added and active molding of nasal cartilage starts. Alar rim is stretched over wide alveolar deformity, and this is elevated into symmetrical and convex form with the help of nasal stent.

The nasal stent is made with 0.036-in stainless steel wire that is secured at the base in the nasal end and this projects into the nostrils. This wire is bent back to make a loop that will hold hard acrylic that supports and on which the tissue is formed. This hard acrylic is lined with soft liner. The upper lobe enters in the nose and lifts the dome until tissue appears blanched. The lower lobe of the stent lifts the apex and defines the top of columella (-Fig. 3 ).

\section{Complications}

- Sometimes misdirected molding of alveolar segments takes place.

- The position of maxilla in the locked-out region will fail to provide support to desired soft tissue, if it is not corrected.

- Sometimes mega nostrils are formed because of overextension of alar sinus on unaffected side.

- Because of loss of retention, molding effect of the appliance is lost. Hence motivation and education of parents are paramount.

- There will be slow progress of closure of cleft if improper and less aggressive tapes are used.

- Sometimes during active intraoral molding, due to application of pressure in primary tooth bud may be exposed.

\section{Benefits}

PNAM appliance has following benefits:

- Tissues are well aligned before surgery and lead to better outcome with less scar formation.

- The long-term benefit is stable nasal shape and forms with less scar formation. ${ }^{4}$

\section{Limitations}

This technique of presurgical nasoalveolar molding also has drawbacks. The process should begin as early as possible; otherwise, the plasticity of both soft and hard tissues reduces and molding becomes difficult. Parents' cooperation is necessary for better prognosis of the treatment.

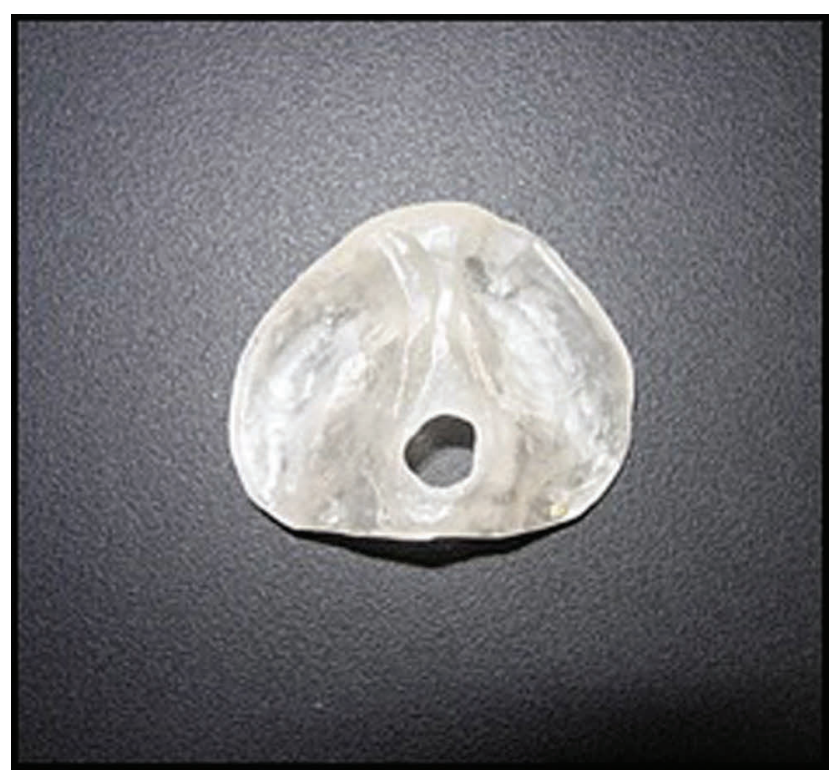

Fig. 3 Prosthesis fabricated. 


\section{Discussion}

Since the introduction of its use in the 1990s, it has become a noninvasive option to improve nasal symmetry before primary repair of a cleft lip. To monitor the progress of individual treatments is important so that the timing for the specific therapeutic steps can be calculated. The goal of treatment in cleft lip and palate patient is to achieve normal anatomy. PNAM is an emerging procedure that leads to tissue expansion and correct the nasal deformity nonsurgically and columellar length deficiency is resolved and with minimal surgery malposition of alveolar segment is corrected. Earlier, the presurgical infant orthopedic (PSIO) has been used to reduce the size of alveolus and palate. However, this appliance only moves the alveolar segments and does not help in repositioning of the deformed surrounding soft tissues such as nasal dome and columella-philtrum region. However, PNAM induces molding of alveolar segment actively and also supports and molds the deformity in nasal cartilages, correction and proper orientation of nasal tip, and also helps in deficient columella lengthening on the cleft side before the initial reparative lip and nasal surgery. Nasal stents enter the nasal aperture and serve as customized tissue expanders that progressively and slowly help in correction of nasal deformity and more precise midline placement of columella. Each step in PNAM procedure is important. It is recommended that the impression should be taken very carefully. Impression of the cleft-lip-palate-nose complex is made shortly after birth within 3 to 4 weeks. Alginate impression material is preferred over impression materials such as condensation silicon. Alginate has advantages such as low cost, easy manipulation, and easy use, but it has poor dimensional stability and low tear resistance.

Technique of prenasoalveolar has many benefits in treating patients with cleft lip and palate deformity. By using this technique, the surgeon is able to achieve better results as there is proper alignment of the lip, alveolus, and nose. Before doing surgery, if approximation of alveolar process is done, it will ease the surgeon to perform gingivoperiosteoplasty successfully. In long-term studies of prenasoalveolar surgical molding, better results are seen as there are less scar tissue formation and better nasal and lip form. ${ }^{6}$ With the help of this, it can further prevent the number of surgeries the patient has to undergo. There is less chance of oronasal fistula and nasal and labial deformities. ${ }^{7}$ As the alveolar segments are in better position and there are increased bony bridges, permanent teeth have better chance of eruption in good position with good periodontal support. ${ }^{8}$ Studies have shown that $60 \%$ of patients who undergo prenasoalveolar surgical molding and gingivoperiosteoplasty do not require secondary bone grafting. ${ }^{9}$

\section{Conflict of Interest}

None declared.

\section{References}

1 Kamble VD, Parkhedkar RD, Sarin SP, Patil PG, Kothari B. Simplifying cleft surgery by presurgical nasoalveolar molding (PNAM) for infant born with unilateral cleft lip, alveolus, and palate: a clinical report. J Prosthodont Res 2013;57(3):224-231

2 Jayashree M, Paul S. Naso alveolar molding in early management of cleft lip and palate. J Indian Prosthodont Soc 2013;13(3):362-365

3 Mitsuyoshi I, Masahiko W, Masayuki F. Simple modified preoperative nasoalveolar moulding in infants with unilateral cleft lip and palate. Br J Oral Maxillofac Surg 2004;42(6):578-580

4 Grayson BH, Maull D. Nasoalveolar molding for infants born with clefts of the lip, alveolus, and palate. Clin Plast Surg 2004;31(2):149-158, vii

5 Grayson BH, Shetye PR. Presurgical nasoalveolar moulding treatment in cleft lip and palate patients. Indian J Plast Surg 2009;42(Suppl):S56-S61

6 Maull DJ, Grayson BH, Cutting CB, et al. Long-term effects of nasoalveolar molding on three-dimensional nasal shape in unilateral clefts. Cleft Palate Craniofac J 1999;36(5):391-397

7 Sato Y, Grayson BH, Cutting CB. Unilateral Cleft Lip and Palate Patients Following Gingivoperiosteoplasty. 99th edition; San Diego, CA: American Association of Orthodontist; 1999

8 Lee C, Grayson BH, Cutting CB. The Effect of Gingivoperiosteoplasty on the Outcome of Secondary Alveolar Bone Graft. 2nd edition; Seattle, Washington: American Cleft Palate Craniofacial Association; 2002

9 Santiago PE, Grayson BH, Cutting CB, Gianoutsos MP, Brecht LE, Kwon SM. Reduced need for alveolar bone grafting by presurgical orthopedics and primary gingivoperiosteoplasty. Cleft Palate Craniofac J 1998;35(1):77-80 